Bull. Austral. Math. Soc.

VoL. 56 (1997) [489-497]

\title{
ON THE INDEX OF DIRAC OPERATORS ON ARITHMETIC QUOTIENTS
}

\begin{abstract}
ANTON DEITMAR
The aim of this note is to show how the trace formula of Arthur-Selberg can be used to derive index theorems for noncompact arithmetic manifolds. Of special interest is the question, under which circumstances there is an index formula without error term, that is, of the same shape as in the compact case. We shall thus present evidence for the hypothesis that the error term for the Euler operator vanishes in the case that the rational rank is smaller than the real rank.
\end{abstract}

\section{INTRODUCTION}

Index theorems for noncompact but finite volume locally symmetric spaces $Y$ usually are of the form

$$
\operatorname{ind}(D)=\int_{Y} \omega+\text { "error terms", }
$$

where $\omega$ is the local index form of the elliptic differential operator $D$. The error terms are associated to the cusps.

Hirzebruch [11] used resolution of cusp-singularities to show that in the case of Hilbert modular surfaces and their signature operators the error terms can be given as special values of $L$-functions. Using the Selberg trace formula, this was extended to higher dimensional Hilbert modular varieties by Müller [14]. Also by means of the Selberg trace formula, Barbasch and Moscovici [5] showed index theorems for real rank one spaces. A detailed analysis of the geometry of arithmetic quotient varieties led Stern $[\mathbf{1 6}, \mathbf{1 7}]$ to index theorems of the above type. By means of the adelic trace formula Labesse [12] investigated the index of the signature operator, focussing on the representation theoretic aspects.

In this note we shall use Arthur's formula [1] to give a geometric index formula of the above type for Dirac operators. The advantage of this index formula is that in some cases like products of real hyperbolic spaces the vanishing of the error term can be read off. The most important example for this is the Euler operator $D^{e}=d+d^{*}$ as an operator from even to odd forms.

In the compact case one has

$$
\text { ind } D^{e}=\int_{Y} \omega=\chi(Y),
$$

Received 18th February, 1997.

Copyright Clearance Centre, Inc. Serial-fee code: 0004-9729/97 \$A2.00+0.00. 
where $\omega$ is the Euler form and $\chi(Y)$ the topological Euler characteristic. The first equality is the Atiyah-Singer index theorem, the second the Gauss-Bonnet equality. In the case of arithmetic quotients Harder [8] has shown that the second equality also holds in the noncompact case. One might wonder whether the first also carries over to noncompact arithmetic quotients. We shall show that the first equality holds in the case of products of rank one spaces modulo arithmetic groups coming from totally real number fields with at least two real embeddings. This contains the case of Hilbert modular varieties. The same assertion is known to be false in general. It seems likely it depends on the condition that the $\mathbb{Q}$-rank is smaller than the $\mathbb{R}$-rank.

\section{Homogeneous DiraC operators AND THEIR INDEX KeRnelS}

An arithmetic quotient is a quotient manifold $X_{\Gamma}=\Gamma \backslash X$ of a globally symmetric space $X$ by a torsion-free arithmetic group $\Gamma$. The space $X$ is assumed not to have compact or Euclidean factors. Under these circumstances the space $X$ can be written as a homogeneous space $X=G / K$, where $G$ is a semisimple real reductive group acting transitively by isometries and $K$ is a maximal compact subgroup of $G$. The group $\Gamma$ is an arithmetic subgroup of $G$.

To be able to use adelic methods we shall further assume that $\Gamma$ is a congruence subgroup, that is, there is a semisimple linear algebraic group $\mathcal{G}$ over $\mathbb{Q}$ with $G=\mathcal{G}(\mathbb{R})$, a compact open subgroup $K_{\Gamma}$ of $\mathcal{G}\left(\mathbb{A}_{f i n}\right)$, where $\mathbb{A}_{f i n}$ is the ring of finite adeles over $\mathbb{Q}$, such that $\Gamma=\mathcal{G}(\mathbb{Q}) \cap K_{\Gamma}$.

In order to have strong approximation available we shall also assume the group $\mathcal{G}$ to be simply connected.

Since the indices of homogeneous Dirac operators are known to vanish otherwise, we shall assume

$$
\operatorname{rank} G=\operatorname{rank} K \text {. }
$$

On the group $\mathcal{G}(\mathbb{A})$ we have a unique Haar measure given by a rational top differential form. This measure is also called the Tamagawa measure. We shall distribute the Tamagawa measure to the factors $\mathcal{G}(\mathbb{R})$ and $\mathcal{G}\left(\mathbb{A}_{\text {fin }}\right)$ in such a way that on $G=\mathcal{G}(\mathbb{R})$ we have the Euler-Poincaré measure given by

$$
\operatorname{vol}(\Gamma \backslash G)=(-1)^{\operatorname{dim} X / 2} \chi\left(X_{\Gamma}\right)
$$

where $\chi$ denotes the Euler-Poincare characteristic. It suffices to insist that this formula holds for cocompact torsion free lattices $\Gamma$, but for arithmetic ones which are not cocompact it holds as well [8].

Let $\mathfrak{g}_{0}=\mathfrak{k}_{0} \oplus \mathfrak{p}_{0}$ denote the polar decomposition of the real Lie algebra $\mathfrak{g}_{0}$ of $G$ where $k_{0}:=\operatorname{Lie}_{\mathbb{R}} K$ and $\mathfrak{p}_{0}$ is the orthogonal complement of $\mathfrak{k}_{0}$ with respect to the Killing form $B$ of $\mathfrak{g}_{0}$. Let $\mathfrak{g}=\mathfrak{k} \oplus \mathfrak{p}$ denote the complexified version. Since $G$ and $K$ have the 
same rank there is a Cartan subgroup $T$ of $G$ which is contained in $K$. Let $\mathfrak{t}$ denote the complex Lie algebra of $T$. Choose an ordering on the root system $\Phi(\mathfrak{g}, \mathfrak{t})$. Since $\mathfrak{p}$ is stable under $\operatorname{Ad}(T)$ it follows that this choice induces a decomposition $\mathfrak{p}=\mathfrak{p}_{+} \oplus \mathfrak{p}_{-}$ according to positive and negative root spaces. This decomposition is a polarisation of the quadratic space $(B, \mathfrak{p})$ and thus the space $S:=\wedge^{*} \mathfrak{p}_{-}$becomes a module under the Clifford algebra $C l(B, \mathfrak{p})$. Since $\mathcal{G}$ is simply connected the homomorphism $K \rightarrow \mathrm{SO}(\mathfrak{p})$ given by the adjoint action factors over the spin group $\operatorname{Spin}(\mathfrak{p}) \subset C l(B, \mathfrak{p})$. So $K$ acts on $S$. The same applies to $\wedge^{*} \mathfrak{p}_{+}$and the $K$-action on the space $\wedge^{*} \mathfrak{p}_{-} \otimes \wedge^{*} \mathfrak{p}_{+}=\wedge^{*} \mathfrak{p}$ coincides with the adjoint action.

The action of $K$ on $S$ leaves invariant the subspaces

$$
S^{+}:=\wedge^{\text {even }} \mathfrak{p}_{-}, \quad S^{-}:=\wedge^{\text {odd }} \mathfrak{p}_{-} .
$$

Let $\left(\tau, V_{\tau}\right)$ be an irreducible unitary representation of $K$ and write $\left(\breve{\tau}, V_{\breve{\tau}}\right)$ for the dual representation. Then the $K$-representations $S^{ \pm} \otimes \tau$ define homogeneous vector bundles $E\left(S^{ \pm} \otimes \tau\right)$ over $X$ whose smooth sections can by identified with the $K$-invariants:

$$
\left(C^{\infty}(G) \otimes S^{ \pm} \otimes \tau\right)^{K}
$$

where $K$ acts on $C^{\infty}(G)$ by right shifts. The same applies to $S$ and we have $E(S \otimes \tau)=$ $E\left(S^{-} \otimes \tau\right) \oplus E\left(S^{+} \otimes \tau\right)$. Let the Lie algebra $g$ act on $C^{\infty}(G)$ by left invariant vector fields, that is, $X f(g):=\left.\frac{d}{d t}\right|_{t=0} f(g \exp (t X))$ for $X \in \mathfrak{g}$ and $f \in C^{\infty}(G)$. Let $\left(X_{j}\right)_{1 \leqslant j \leqslant \operatorname{dim} X}$ denote an orthonormal basis of $\mathfrak{p}$ the we have the Dirac operator acting on $C^{\infty}(E(S \otimes \tau))$ :

$$
D_{\tau}:=\sum_{j=0}^{\operatorname{dim} X} X_{j} \otimes c\left(X_{j}\right) \otimes 1,
$$

where we have written $c\left(X_{j}\right)$ for the Clifford action of $X_{j} \in \mathfrak{p} \subset C l(B, \mathfrak{p})$ on $S$. Clearly $D_{\tau}$ commutes $C^{\infty}\left(E\left(S^{+} \otimes \tau\right)\right)$ and $C^{\infty}\left(E\left(S^{-} \otimes \tau\right)\right)$ and we shall write $D_{\tau}^{ \pm}$for the restriction of $D_{\tau}$ to $C^{\infty}\left(E\left(S^{ \pm} \otimes \tau\right)\right)$. Then $D_{\tau}^{+}$and $D_{\tau}^{-}$are adjoints of each other.

The homogeneous bundle $E(S \otimes \tau)$ pushes down to a bundle $E_{\Gamma}(S \otimes \tau)$ over $X_{\Gamma}$ whose space of smooth sections can be identified with $\left(C^{\infty}(\Gamma \backslash G) \otimes S \otimes \tau\right)^{K}$.

In $[13]$ it is proven that there is a compactly supported smooth function $g_{\tau}$ such that

$$
\operatorname{tr} \pi\left(g_{\tau}\right)=\operatorname{dim}\left(V_{\pi} \otimes S^{+} \otimes V_{\ddot{*}}\right)^{K}-\operatorname{dim}\left(V_{\pi} \otimes S^{-} \otimes V_{*}\right)^{K}
$$

for all $\pi \in \hat{G}$.

We want to show that $\operatorname{tr} \pi\left(g_{\tau}\right)$ vanishes for a principal series representation $\pi$. To this end let $P=$ MAN be a parabolic subgroup with $A \subset \exp (\mathfrak{p})$. Let $\left(\xi, V_{\xi}\right)$ denote an irreducible unitary representation of $M$ and $e^{\nu}$ a quasicharacter of $A$. Let $\pi_{\xi, \nu}:=$ $\operatorname{Ind}_{P}^{G} \xi \otimes e^{\nu+\rho_{P}} \otimes 1$. 
LEMMA 1.1. We have $\operatorname{tr} \pi_{\xi, \nu}\left(g_{\tau}\right)=0$.

PROOF: By Frobenius reciprocity we have

$$
\operatorname{Hom}_{K}\left(\gamma,\left.\pi_{\xi, \nu}\right|_{K}\right) \cong \operatorname{Hom}_{K_{M}}\left(\left.\gamma\right|_{K_{M}}, \xi\right)
$$

where $K_{M}:=K \cap M$. The claim will follow from $\left.\left.S^{+}\right|_{K_{M}} \cong S^{-}\right|_{K_{M}}$. To prove this let $0 \neq \omega \in \mathfrak{p}_{-}$be in the image of the projection of $\mathfrak{a}:=\operatorname{Lie} A$ to $\mathfrak{p}_{-}$. Then $K_{M}$ acts trivially

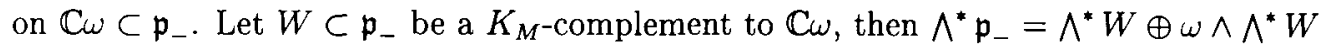
and so $S^{+}=\wedge^{\text {even }} W \oplus \omega \wedge \wedge^{\text {odd }} W \cong{ }_{K_{M}} \wedge^{\text {odd }} W \oplus \omega \wedge \wedge^{\text {even }} W=S^{-}$.

For any unitary representation $\pi$ of $G$ we define the Dirac operator

$$
D_{\tau, \pi}:=\sum_{j=0}^{\operatorname{dim} X} \pi\left(X_{j}\right) \otimes c\left(X_{j}\right) \otimes 1
$$

acting on $\left(\pi^{\infty} \otimes S \otimes \tau\right)^{K}$. Write $D_{\tau, \pi}^{ \pm}$for the restriction to $\left(\pi^{\infty} \otimes S^{ \pm} \otimes \tau\right)^{K}$. If ker $D_{\tau, \pi}^{+}$ and $\operatorname{ker} D_{\tau, \pi}^{-}$are finite dimensional we define

$$
\text { ind } D_{\tau, \pi}^{+}:=\operatorname{dim} \operatorname{ker} D_{\tau, \pi}^{+}-\operatorname{dim} \operatorname{ker} D_{\tau, \pi}^{-} \text {. }
$$

LEMma 1.2. For $\pi \in \widehat{G}$ the kernel of $D_{\tau, \pi}$ is finite dimensional and we have

$$
\text { ind } D_{\tau, \pi}^{+}=\operatorname{tr} \pi\left(g_{\breve{\tau}}\right) \text {. }
$$

Proof: Since $K$-types have finite multiplicities in $\pi$ it follows that $\left(\pi^{\infty} \otimes S \otimes \tau\right)^{K}$ is finite dimensional. On this finite dimensional space the operators $D_{\tau, \pi}^{+}$and $D_{\tau, \pi}^{-}$are adjoints of each other, so

$$
\text { ind } \begin{aligned}
D_{\tau, \pi}^{+} & =\operatorname{dim} \operatorname{ker} D_{\tau, \pi}^{-} D_{\tau, \pi}^{+}-\operatorname{dim} \operatorname{ker} D_{\tau, \pi}^{+} D_{\tau, \pi}^{-} \\
& =\left.\operatorname{dim} \operatorname{ker} D_{\tau, \pi}^{2}\right|_{\left(\pi^{\infty} \otimes S^{+} \otimes \tau\right)}-\left.\operatorname{dim} \operatorname{ker} D_{\tau, \pi}^{2}\right|_{\left(\pi^{\infty} \otimes S^{-} \otimes \tau\right)} .
\end{aligned}
$$

The formula of Parthasarathy [15] (see also [3]) implies

$$
D_{\tau, \pi}^{2}=-\pi(C)+\tau\left(C_{K}\right)+B\left(\rho_{K}\right)-\left.B(\rho)\right|_{\left(\pi^{\infty} \otimes S \otimes \tau\right)^{K}},
$$

where $C$ and $C_{K}$ are the Casimir operators of $G$ and $K$.

This gives the claim.

The Dirac operator for the $G$-representation on $L^{2}(\Gamma \backslash G)$ will be denoted $D_{\tau, \Gamma}$. Recall that $L^{2}(\Gamma \backslash G)$ decomposes as

$$
L^{2}(\Gamma \backslash G)=L^{2}(\Gamma \backslash G)_{d i s c} \oplus L^{2}(\Gamma \backslash G)_{c o n t},
$$

where $L^{2}(\Gamma \backslash G)_{d i s c}$, the discrete part, is the sum of all irreducible subrepresentations of $L^{2}(\Gamma \backslash G)$ and $L^{2}(\Gamma \backslash G)_{\text {cont }}$ is a continuous Hilbert integral extended over the principal series. Let $R\left(g_{\tau}\right)$ denote the convolution operator $\varphi \mapsto \varphi * \breve{g}_{\tau}$, where $\breve{g}_{\tau}(x):=g_{\tau}\left(x^{-1}\right)$. Then $R\left(g_{\tau}\right)=R_{\text {cont }}\left(g_{\tau}\right)+R_{\text {disc }}\left(g_{\tau}\right)$ and [1, Theorem 7.1] says that $R_{\text {disc }}\left(g_{\tau}\right)$ is a trace class operator. The formula of Parthasarathy above fixes the value $\pi(C)$ when $D_{\tau, \pi}^{+}$has a non zero kernel. This gives: 
LEMMA 1.3. The spaces $\operatorname{ker} D_{\tau, \Gamma}^{+} \cap L^{2}(\Gamma \backslash G)$ and $\operatorname{ker} D_{\tau, \Gamma}^{-} \cap L^{2}(\Gamma \backslash G)$ are finite dimensional. Denote the difference of their dimensions by ind $D_{\tau, \Gamma}^{+}$then

$$
\text { ind } D_{\tau, \Gamma}^{+}=\operatorname{tr}\left(g_{*} \mid L^{2}(\Gamma \backslash G)_{d i s c}\right) .
$$

The association $\tau \mapsto g_{\tau}$ extends to virtual representations by linearity. Consider the virtual representation of $K$ on $S^{+}-S^{-}$. We define

$$
f_{\tau}:=g_{\tau \otimes\left(S^{+}-S^{-}\right)} .
$$

It follows that for $\pi \in \hat{G}$ we have

$$
\operatorname{tr} \pi\left(f_{\tau}\right)=\sum_{q=0}^{\operatorname{dim} X}(-1)^{q} \operatorname{dim}\left(V_{\pi} \otimes \wedge^{q} \mathfrak{p} \otimes V_{\breve{\tau}}\right)^{K} .
$$

An element $g$ of $G$ is called elliptic if it lies in a compact subgroup of $G$. For any $g \in G$ and a compactly supported smooth function $f$ on $G$ let

$$
\mathcal{O}_{g}(f):=\int_{G / G_{g}} f\left(x g x^{-1}\right) d x
$$

denote the orbital integral. The required normalisation of Haar measures of $G$ and $G_{g}$ follows [10].

Proposition 1.4. Let $g$ be a semisimple element of the group $G$. If $g$ is not elliptic, the orbital integrals $\mathcal{O}_{g}\left(f_{r}\right)$ and $\mathcal{O}_{g}\left(g_{\tau}\right)$ vanish. If $g$ is elliptic we may assume $g \in T$, where $T$ is a Cartan in $K$ and then we have

$$
\mathcal{O}_{g}\left(f_{\tau}\right)=\frac{\operatorname{tr} \tau(g)\left|W\left(\mathfrak{t}, \mathfrak{g}_{g}\right)\right| \prod_{\alpha \in \Phi_{g}^{+}}\left(\rho_{g}, \alpha\right)}{\left[G_{g}: G_{g}^{0}\right] c_{g}},
$$

for all elliptic $g$ and

$$
\mathcal{O}_{g}\left(g_{\tau}\right)=\frac{\operatorname{tr} \tau(g)}{\operatorname{det}\left(1-g^{-1} \mid \mathfrak{p}_{+}\right)},
$$

if $g$ is regular elliptic. For general elliptic $g$ we have

$$
\mathcal{O}_{g}\left(g_{\tau}\right)=\frac{\sum_{s \in W(T, K)} \operatorname{det}(s) \widetilde{\omega}_{g} g^{s \lambda_{\tau}+\rho-\rho_{K}}}{\left[G_{g}: G_{g}^{0}\right] c_{g} g^{\rho} \prod_{\alpha \in \phi^{+}-\phi_{g}^{+}}\left(1-g^{-\alpha}\right)},
$$

where $c_{g}$ is Harish-Chandra's constant, it does only depend on the centraliser $G_{g}$ of $g$. Its value is given in [7], further $\tilde{\omega}_{\gamma}$ is the differential operator as in $[\mathbf{9}, \mathrm{p} .33]$.

If $G$ is a product of real rank one groups then the orbital integral $\mathcal{O}_{g}\left(f_{\tau}\right)$ vanishes also for $g$ non-semisimple. 
ProOF: The computation of the orbital integrals of semisimple elements is essentially in [7]. For the non-semisimple elements it suffices to assume that the real rank of $G$ is one. Consider a non-semisimple element $g$. By [4, Section 6], we get a curve $t \mapsto z_{t}$ of semisimple elements and a natural number $m$ such that $\mathcal{O}_{g}\left(f_{\tau}\right)=\lim _{t \rightarrow 0} t^{m / 2} \mathcal{O}_{z_{t}}\left(f_{\tau}\right)$. The function $x \mapsto \mathcal{O}_{x}\left(f_{\tau}\right)$ is bounded on semisimple elements by the proposition and therefore we have $\mathcal{O}_{g}\left(f_{\tau}\right)=0$ for $g$ non semisimple.

Conjecture 1.5. The orbital integral $\mathcal{O}_{g}\left(f_{\tau}\right)$ vanishes for all $g$ and all groups $G$.

\section{THE $G$-INDEX}

Let $\mathcal{R}_{G} \subset B\left(L^{2}(G)\right)$ denote the von Neumann algebra defined as the commutant of the left representation of $G$ on $L^{2}(G)$. On $\mathcal{R}_{G}$ there is a canonical faithful, normal, semi-finite trace $\operatorname{tr}_{G}$, called the $G$-trace, uniquely determined by the property that

$$
\operatorname{tr}_{G}\left(R(f)^{*} R(f)\right)=\int_{G}|f(g)|^{2} d g,
$$

where $R$ denotes the right representation of $G$ (See [6].) Let $\operatorname{dim}_{G}$ denote the dimension defined by the $G$-trace.

Choose a compact form $G^{d}$ of $G$ in a way that $G^{d}$ contains the compact group $K$. The homogeneous space $X^{d}=G^{d} / K$ then is symmetric and is called the dual symmetric space to $X$.

Let $\lambda=\lambda_{\tau}$ denote the infinitesimal character of $\tau$ then $\lambda$ also defines an infinitesimal character of some irreducible representation $W_{\lambda}$ of $G^{d}$.

PROPOSITION 2.1. The spaces $\operatorname{ker} D_{\tau}^{ \pm} \cap L^{2}\left(E\left(S^{ \pm} \otimes \tau\right)\right)$ are finite-dimensional under $\operatorname{dim}_{G}$. Let $\operatorname{ind}_{G}\left(D_{\tau}^{+}\right)$denote the $G$-index of $D_{\tau}^{+}$, which is, by definition, the difference of these two $G$-dimensions. Then if $\lambda$ is regular with respect to the full root system we have

$$
\operatorname{ind}_{G}\left(D_{\tau}^{+}\right)=\frac{\operatorname{dim} W_{\lambda}}{\chi\left(X^{d}\right)}
$$

where $\chi\left(X^{d}\right)$ is the Euler-characteristic of $X^{d}$. If $\lambda$ is not regular, the $G$-index of $D_{\tau}^{+}$ vanishes.

It is known that the Euler characteristic is positive and that $\chi\left(X^{d}\right)=\left|W\left(T, G^{d}\right)\right|$, where $T$ is a Cartan subgroup of $G^{d}$.

PRoOF: The finite dimensionality is [6, Lemma 3.2]. The index formula follows from formulas (3.7)-(3.13) of [3].

\section{THE INDEX THEOREM}

Assume now that $\mathcal{G}$ is the restriction to $\mathbb{Q}$ of some algebraic group $\mathcal{H}$ over a number field $F$. It is known that the index of the Dirac operator $D_{\tau, \Gamma}$ vanishes if $\operatorname{rank} G>\operatorname{rank} K$. 
If $F$ has a complex place then it follows that $\operatorname{rank} G>\operatorname{rank} K$. So, in order to have a nontrivial theory we shall assume the field $F$ to be totally real. Let $S$ denote the set of Archimedian places of $F$. We assume that $S$ has at least two elements.

THEOREM 3.1. The index of the operator $D_{\tau, \Gamma}^{+}$is given by

$$
\text { ind }\left(D_{\tau, \Gamma}^{+}\right)=\operatorname{ind}_{G}\left(D_{\tau}^{+}\right) \chi\left(X_{\Gamma}\right)+R(\tau, \Gamma) \text {, }
$$

where the "error term" $R(\tau, \Gamma)$ equals

$$
R(\tau, \Gamma)=\sum_{\gamma \in \Gamma_{n s} / G} a(\gamma) \mathcal{O}_{\gamma}\left(g_{\tau}\right) .
$$

The sum is extended over the set of non-semisimple elements $\Gamma_{n s}$ of $\Gamma$ modulo the equivalence relation $(G, S)$ defined in $[\mathbf{1}]$. The constant $a(\gamma)$ coincides with $a^{G}(S, \gamma)$ of $[\mathbf{1}]$ up to a volume factor.

In the case $G=\operatorname{Res}_{F / G} S O(n, 1)$ for $n \geqslant 3$, the error term vanishes. Here $S O(n, 1)$ stands for the special orthogonal group of a quadratic form which has signature $(n, 1)$ over the reals.

Note that the first summand on the right hand side of the index formula also coincides with the integral $\int_{X_{\Gamma}} \omega$, where $\omega$ is the index form of $D_{\tau}$. This also equals the $\Gamma$-index [2] of $D_{\tau}^{+}$, so we have ind $\left(D_{\tau, \Gamma}^{+}\right)=\operatorname{ind}_{\Gamma}\left(D_{\tau}^{+}\right)+R(\tau, \Gamma)$.

ProOF: Let $K_{\Gamma}$ be the compact open subgroup of $\mathcal{G}\left(\mathbb{A}_{f i n}\right)$ such that $\Gamma=K_{\Gamma} \cap \mathcal{G}(\mathbb{Q})$. Define a compactly supported function $f$ on $\mathcal{G}(\mathbb{A})$ by $f=f_{\text {fin }} \otimes f_{\infty}$, where $f_{\text {fin }}:=$ $\left(1 / \operatorname{vol}\left(K_{\Gamma}\right)\right) 1_{K_{\Gamma}}$ and $f_{\infty}:=g_{\tau}$. Plug the function $f$ into [1, Theorem 7.1(b)]. Then use Lemma 1.3 and Proposition 1.4.

For the last assertion recall that Proposition 1.4 and [4; Section 6] imply that $R(\tau, \Gamma)=0$ if $K$ has discrete center. The latter condition is satisfied for $S O(n, 1)$.

\section{The Euler OPERATOR}

Consider the homogeneous vector bundle $E=E(\tau)$ associated with the representation $\tau$. Choose a homogeneous connection on $E(\tau)$. These always exist and in the case that $\tau=\left.\sigma\right|_{K}$, where $\sigma$ is a finite dimensional representation of $G$, there is a unique flat homogeneous connection. This is the case considered in [17]. The choice of a connection gives us an exterior differential

$$
d: \Omega(E) \rightarrow \Omega^{+1}(E) .
$$

The representation $\tau$ being unitary gives us a homogeneous Hermitian metric on $E$ and we can define the formal adjoint $d^{*}$ of $d$ and the Euler operator:

$$
D_{\tau}^{e}:=d+d^{*}: \Omega^{\text {even }}(E) \rightarrow \Omega^{\text {odd }}(E) .
$$

We now come to the main result of this note: 
THEOREM 4.1. Assume that $G=\operatorname{Res}_{F / \mathbb{Q}} \mathcal{H}$, where $\mathcal{H}$ is a semisimple connected linear algebraic group over the totally real number field $F$. Assume $F$ has at least two real embeddings and over $\mathbb{R}, \mathcal{H}$ is a product of rank one groups. Let $G:=\mathcal{G}(\mathbb{R})$ and put $X=G / K$, the symmetric space attached to $G$. Let $\Gamma$ be a torsion free congruence subgroup of $G$. Write $X_{\Gamma}=\Gamma \backslash X$ for the quotient manifold. Let $E(\tau)$ be a homogeneous vector bundle over $X$ given by a unitary finite dimensional representation $\tau$ of the compact group $K$ and let $D_{\tau, \Gamma}^{e}$ be the Euler operator of the pushdown of $E(\tau)$ to $X_{\Gamma}$. Then $D_{\tau, \Gamma}^{e}$ has a well defined index and

$$
\text { ind }\left(D_{\tau, \Gamma}^{e}\right)=\operatorname{ind}_{G}\left(D_{\tau}^{e}\right) \chi\left(X_{\Gamma}\right)
$$

Assume Conjecture 1.5 holds. Then the condition on the real points of $G$ in the above theorem can be removed.

For the usual Euler operator $D_{\Gamma}^{e}$ on $X_{\Gamma}$ we have

$$
\operatorname{ind}\left(D_{\Gamma}^{e}\right)=\chi\left(X_{\Gamma}\right)
$$

Proof: The index of $D_{\tau, \Gamma}^{e}$ coincides with the index of $D_{\tilde{\tau}, \Gamma}^{+}$, where $\tilde{\tau}$ is the virtual representation $\tau \otimes\left(S^{+}-S^{+}\right)$. With Lemma 1.3 it follows that the index exists. The index theorem tells us that

$$
\text { ind }\left(D_{\tau, \Gamma}^{e}\right)=\operatorname{ind}_{G}\left(D_{\tau}^{e}\right) \chi\left(X_{\Gamma}\right)+R(\widetilde{\tau}, \Gamma)
$$

Proposition 1.4 tells us that $R(\widetilde{\tau}, \Gamma)=0$.

The last assertion is clearly valid for cocompact groups $\Gamma$. This implies that the $G$-index must be one.

\section{REFERENCES}

[1] J. Arthur, 'The invariant trace formula II. Global theory.', J. Amer. Math. Soc. 1 (1988), 501-554.

[2] M. Atiyah, 'Elliptic operators, discrete groups and Von Neumann algebras', in Colloque "Analyse et Topologie" en l'Honneur de Henri Cartan (Orsay, 1974), Astérisque 32-33 (Soc. Math. France, Paris, 1976), pp. 43-72.

[3] M. Atiyah and W. Schmid, 'A geometric construction of the discrete series for semisimple Lie groups', Invent. Math. 42 (1977), 1-62.

[4] D. Barbasch, 'Fourier inversion for unipotent invariant integrals', Trans. Amer. Math. Soc. 249 (1979), 51-83.

[5] D. Barbasch and H. Moscovici, ' $L$-index and the Selberg trace formula', J. Funct. Anal. 53 (1983), 151-201.

[6] A. Connes and H. Moscovici, 'The $L^{2}$-index theorem for homogeneous spaces of Lie groups', Ann. of Math. 115 (1982), 291-330.

[7] A. Deitmar, 'Higher torsion zeta functions', Adv. Math. 110 (1995), 109-128. 
[8] G. Harder, 'A Gauss-Bonnet formula for discrete arithmetically defined groups', Ann. Sci. École Norm. Sup. 4 (1971), 409-455.

[9] Harish-Chandra, 'Discrete series for semisimple Lie groups II', Acta Math. 116 (1966), $1-111$.

[10] Harish-Chandra:, 'Harmonic analysis on real reductive groups I. The theory of the constant term', J. Funct. Anal. 19 (1975), 104-204.

[11] F. Hirzebruch, 'Hilbert modular surfaces', Enseign. Math. 19 (1973), 183-281.

[12] J.P. Labesse, 'Signature des variétés modulaires de Hilbert et representations diédrales', in Cohomology of arithmetic groups and automorphic forms (Luminy-Marseille, 1989), Lecture Notes in Mathematics 1447 (Springer-Verlag, Berlin, Heidelberg, New York, 1990), pp. 249-260.

[13] J.P. Labesse, 'Pseudo-coefficients très cuspidaux et K-théorie', Math. Ann. 291 (1991), 607-616.

[14] W. Müller, 'Signature defects of cusps of Hilbert modular varieties and values of $L$-series at $s=1$ ', J. Differential Geom. 20 (1984), 55-119.

[15] R. Parthasarathy, 'Dirac operators and the discrete series', Ann. of Math. 103 (1976), 375-394.

[16] M. Stern, ' $L^{2}$-index theorems on locally symmetric spaces', Invent. Math. 96 (1989), 231-282.

[17] M. Stern, 'Lefschetz formulae for arithmetic varieties', Invent. Math. 115 (1994), 241-296.

Mathematisches Institut

Universitat Heidelberg

Im Neuenheimer Feld 288

D-69120 Heidelberg

Germany 